\title{
Identification of $\$ 23$ causing both interspecific hybrid male sterility and environment-conditioned male sterility in rice
}

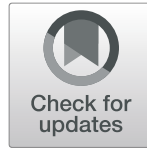

Chaowei Fang ${ }^{1,2+}$, Le Li ${ }^{2,3+}$, Runming He ${ }^{2,4}$, Daiqi Wang ${ }^{1,2}$, Man Wang ${ }^{1,2}$, Qian Hu ${ }^{1,2}$, Qianru Ma ${ }^{1,2}$, Kaiyi Qin ${ }^{1,2}$, Xueye Feng ${ }^{1,2}$, Guiquan Zhang ${ }^{1,2}$, Xuelin Fü ${ }^{2}$ and Ziqiang Liu ${ }^{1,2^{*}}$ (D)

\begin{abstract}
Background: Oryza glumaepatula represents an important resource of genetic diversity that can be used to improve rice production. However, hybrid sterility severely restricts gene flow between Oryza species, and hinders the utilization of distant heterosis in hybrid rice breeding.

Results: In order to fully exploit the beneficial genes of O. glumaepatula and facilitate the conservation of these gene resources, a set of chromosome single-segment substitution lines (SSSLs) was developed using an indica variety HJX74 as the recurrent parent and an accession of $O$. glumaepatula as the donor parent. During the process of SSSLs development, S23, a locus conferring hybrid male sterility between O. sativa and O. glumaepatula, was identified and fine mapped to $11.54 \mathrm{~kb}$ and $7.08 \mathrm{~kb}$ genomic region in $O$. sativa and $O$. glumaepatula, respectively, encoding three and two candidate ORFs, respectively. QRT-PCR and sequence analysis excluded one common ORF as the candidate gene. In addition, hybrid male sterility caused by $\$ 23$ was environment-sensitive, and could be observed only in natural short-day (NSD).

Conclusion: Identification and candidate genes analysis of S23 in this study provides a valuable example to study the crosstalk between interspecific $F_{1}$ hybrid male sterility and environment-conditioned male sterility in rice, facilitates reserving and utilizing favorable genes or alleles of wild Oryza species, and allows for a more efficient exploitation of distant heterosis in hybrid rice breeding.
\end{abstract}

Keywords: Oryza glumaepatula, Chromosome single-segment substitution lines (SSSLs), Interspecific hybrid sterility, Environment-conditioned male sterility, S23

\section{Background}

Agriculture relies heavily on the genetic diversity of crop plants. It is estimated that less than $15 \%$ of the potential diversity has been utilized in cultivated rice (http:// www.fao.org/3/y4751e/y4751e0b.htm\#bm11). The limited genetic diversity of cultivated rice renders them more vulnerable to environment and jeopardizes the potential for sustained genetic improvement over the long term

\footnotetext{
* Correspondence: zqliu@scau.edu.cn

${ }^{+}$Chaowei Fang and Le Li contributed equally to this work.

${ }^{1}$ State Key Laboratory for Conservation and Utilization of Subtropical

Agro-Bioresources, South China Agricultural University, Guangzhou 510642,

China

${ }^{2}$ Guangdong Provincial Key Laboratory of Plant Molecular Breeding, College of Agriculture, South China Agricultural University, Guangzhou 510642, China Full list of author information is available at the end of the article
}

(Tanksley and McCouch 1997). The wild species of the genus Oryza serve as a virtually untapped reservoir of genetic diversity that can be used to improve rice production (Brar and Khush 1997). Therefore, transferring genes that control desirable traits from wild rice to domestic cultivars has proven to be an important strategy in rice breeding (McCouch et al. 2007). Furthermore, hybrids from crosses between species have stronger hybrid vigor and greater yield potential than those within subspecies. However, hybrid sterility, a postzygotic reproductive barrier, is quite common in the hybrid plants which fail to produce fertile pollen or embryo sacs during reproductive development, thus restricting gene flow between Oryza species, maintaining species identity during speciation and hindering the utilization 
of distant heterosis in hybrid rice breeding (Ouyang and Zhang 2013).

Molecular genetic studies over two decades have revealed more than 40 loci causing interspecific or intersubspecific hybrid sterility in rice (Ouyang et al. 2009; Ouyang and Zhang 2013). Major progress has been made recently in understanding molecular mechanisms of hybrid sterility by molecular cloning of causal genes, revealing molecular mechanism of hybrid sterility in rice fitting two genetic models, one-locus sporo-gametophytic interaction model and the duplicate gametic-lethal model (Chen et al. 2008; Long et al. 2008; Yamagata et al. 2010; Mizuta et al. 2010; Yang et al. 2012; Kubo et al. 2016; Yu et al. 2016, 2018; Nguyen et al. 2017; Xie et al. 2017; Shen et al. 2017; Koide et al. 2018). The representative loci for the one-locus sporo-gametophytic model include hybrid female sterility loci S5, S7 and HSA1 (Chen et al. 2008; Yang et al. 2012; Kubo et al. 2016; Yu et al. 2016), and hybrid male sterility loci $S a, S c$ and qHMS7 (Long et al. 2008; Shen et al. 2017; Yu et al. 2018), as well as $S 1$ which confers both hybrid male and female sterility (Xie et al. 2017; Koide et al. 2018). Each locus contains two or more closely located genes interacting to cause gamete sterility, such as three genes at S5 locus functioning in "Killer-Protector" model (Yang et al. 2012), two genes at $S a$ locus functioning in "two gene/three component interaction" model (Long et al. 2008), two genes in qHMS7 functioning in "Toxin-Antidote" model ( $\mathrm{Yu}$ et al. 2018), multiple alleles at $S c$ locus functioning in allelic suppression pattern (Shen et al. 2017), and two genes in HSA1 locus functioning in an allele-specific epistatic interaction pattern (Kubo et al. 2016). Although $S 1$ was identified as a single gene, different groups revealed different causal gene responsible for S1-induced hybrid sterility, suggesting a more complicated molecular mechanism for $S 1$ instead of a simple model of a single gene function (Xie et al. 2017; Koide et al. 2018). In the duplicate gametic-lethal model, gamete-essential genes were duplicated on different chromosomes, followed by reciprocal loss of one of the duplicated genes in the divergent species. Therefore, in the hybrids, genetic segregation and recombination gave rise to gametes with none of the duplicated functional genes, leading to hybrid sterility. In rice, reciprocal loss of duplicated gene pairs, such as $S 27 / S 28, D P L 1 / D P L 2$ and DSG1/DSG2, produces about $25 \%$ sterile pollen grains that lack both of the functional loci in hybrids, thus contributed to reproductive barrier in interspecific or intersubspecific crosses (Yamagata et al. 2010; Mizuta et al. 2010; Nguyen et al. 2017).

In order to fully exploit the beneficial genes of wild Oryza species and facilitate the conservation of these gene resources, we started to develop SSSLs in the genetic background of an indica cultivar HJX74 using accessions from wild Oryza species as donors, and had developed 99 SSSLs carrying donor segments of O. meridionalis ( $\mathrm{He}$ et al. 2017). In the present study, we identified a locus $S 23$ conferring environment-sensitive hybrid male sterility between $O$. sativa and $O$. glumaepatula, another wild Oryza species belonging to AA genome group, during the process of the development of SSSLs using an accession of O. glumaepatula as the donor parent. $S 23$ was fine mapped to $11.54 \mathrm{~kb}$ and 7.08 kb genomic region in O. sativa and O. glumaepatula, respectively, encoding three and two candidate ORFs, respectively. Candidate genes were analyzed and their interaction with environment was discussed. Based on these findings, $S 23$ can be selected as a target for genome editing to break down the reproductive barrier between cultivated rice and wild rice for reserving and utilizing favorable genes or alleles of wild Oryza species, and for facilitating hybrid rice breeding.

\section{Results}

A segregation distortion observed in SSSLs development

The procedure for the development and analysis of SSSL was summarized in Fig. 1. Totally, 168 SSSLs were developed, with the substituted segments covering $81 \%$ of the rice genome (Unpublished data).

In $\mathrm{BC}_{5} \mathrm{~F}_{2}$ generation, NIL7 was detected to have two substituted segments on chromosome 7 and chromosome 12, respectively (Fig. 1 and Additional file 1: Figure S1). Therefore, NIL7 was crossed with HJX74 in order to develop two SSSLs with only one substituted segment for each SSSL. Under NSD condition, two hundred and twenty-one $\mathrm{F}_{2}$ plants were genotyped using 5 SSR (Simple Sequence Repeats) markers (RM47, M429, M22060, PSM148 and M22172) on the substituted segment of chromosome 7 and 2 SSR markers (RM17, MM2968) on the substituted segment of chromosome 12. A segregation distortion of the genotype was found for the 5 SSR markers on chromosome 7. The homozygous genotype for O. glumaepatula was significantly lower than the expected $25 \%$, suggesting that the gametes (male or female) derived from O. glumaepatula were selectively aborted and were not transmissible to the progeny. In contrast, the segregation of the 2 SSR markers on chromosome 12 was normal, fitting the 1:2:1 ratio (Table 1). Our results indicated the substituted segment on chromosome 7 contained a gene locus controlling hybrid sterility between $O$. sativa and O. glumaepatula.

\section{Identification of $S 23$ conferring hybrid male sterility}

SSSL-S23, a SSSL with only the substituted segment on chromosome 7 selected from the $F_{2}$ population generated from crossing NIL7 with HJX74, was further crossed with 


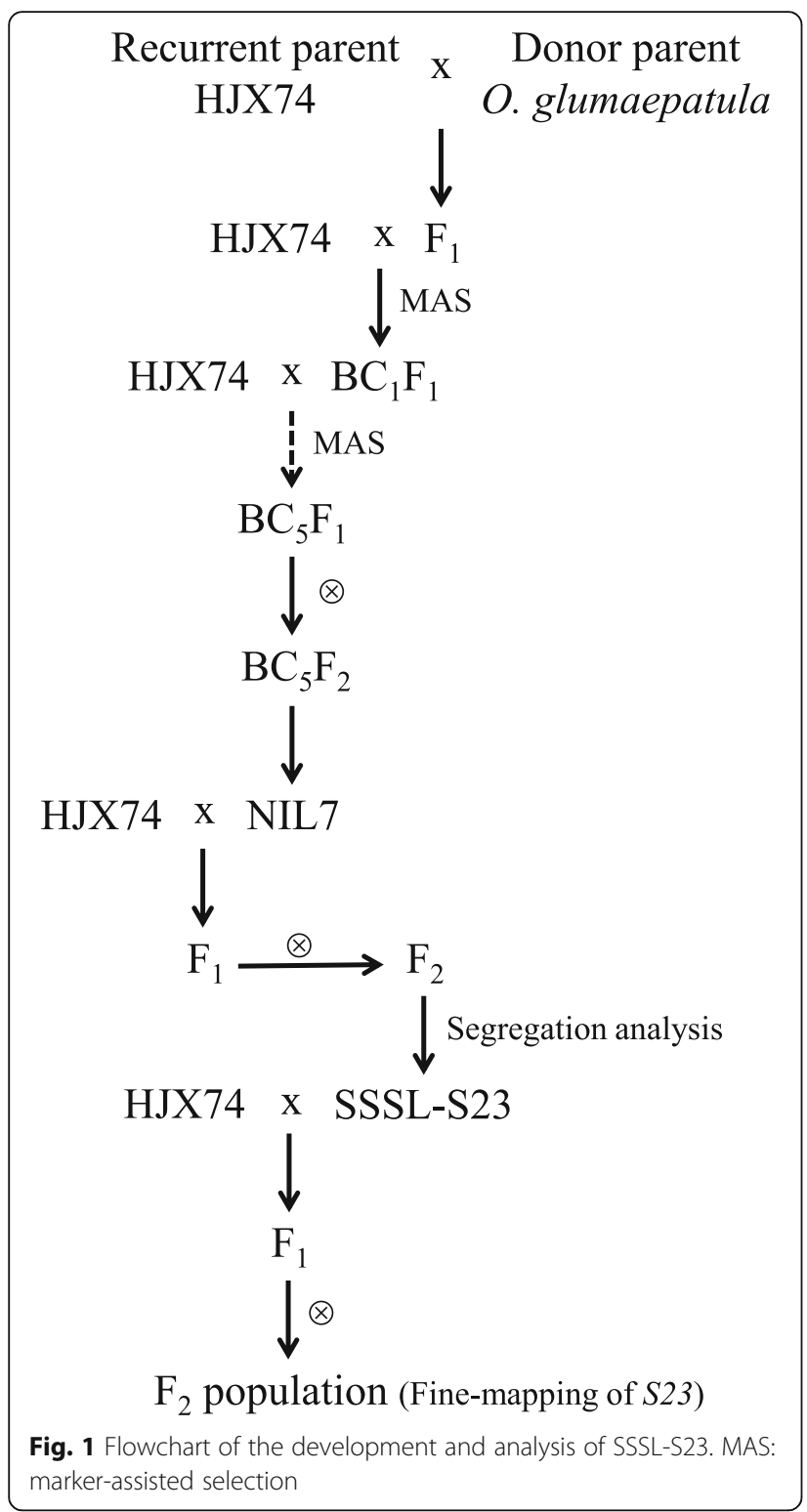

Table 1 Segregation analysis of the $F_{2}$ families derived from crossing NIL7 with HJX74

\begin{tabular}{|c|c|c|c|c|c|c|}
\hline \multirow[t]{2}{*}{ Chromosome } & \multirow{2}{*}{$\begin{array}{l}\text { Molecular } \\
\text { marker }\end{array}$} & \multicolumn{3}{|c|}{ No. of $F_{2}$ plants } & \multirow[t]{2}{*}{$x^{2}(1: 2: 1)$} & \multirow[t]{2}{*}{$P$ value } \\
\hline & & $\mathrm{H} / \mathrm{H}$ & $\mathrm{H} / \mathrm{G}$ & $\mathrm{G} / \mathrm{G}$ & & \\
\hline \multirow[t]{5}{*}{7} & RM47 & 85 & 113 & 13 & 50.20 & $5.51 \mathrm{E}-15^{* * *}$ \\
\hline & M429 & 86 & 118 & 7 & 67.39 & $3.24 \mathrm{E}-14^{* * *}$ \\
\hline & M22060 & 89 & 116 & 6 & 62.12 & $2.33 \mathrm{E}-15^{* * *}$ \\
\hline & PSM148 & 90 & 109 & 12 & 57.90 & $2.67 \mathrm{E}-13^{* * *}$ \\
\hline & M22172 & 81 & 109 & 20 & 35.58 & $1.73 \mathrm{E}-08^{* * *}$ \\
\hline \multirow[t]{2}{*}{12} & RM17 & 54 & 97 & 58 & 1.24 & 0.541 \\
\hline & MM2968 & 53 & 97 & 59 & 1.43 & 0.491 \\
\hline
\end{tabular}

Note: $\mathrm{H} / \mathrm{H}, \mathrm{G} / \mathrm{G}$ and $\mathrm{H} / \mathrm{G}$ represented the homozygous genotype for $\mathrm{HJX74,O}$. gulmaepatula and the heterozygous genotype, respectively, at the corresponding molecular markers. $P$ value was determined with a Student's $t-$ test analysis. ${ }^{* * *}$ represented significance at $P=0.001$
HJX74 to generate $F_{1}$ and $F_{2}$ population for analyzing hybrid sterility locus (Fig. 1). Under NSD condition, the pollen stainability conducted between the parents and the $\mathrm{F}_{1}$ hybrid under microscope showed that the pollen fertility of parents HJX74 and SSSL-S23 were normal (Table 2, Fig. $2 a$ and $b$ ), whereas their $F_{1}$ hybrid showed clear pollen sterility (Table 2 and Fig. 2c), and the type of pollen abortion was stained abortion (Fig. 2c). Although the pollen sterility was observed, the anther developed normal in $\mathrm{F}_{1}$ hybrid (Fig. 2a-c). The spikelet fertility of $\mathrm{F}_{1}$ hybrid was $89.61 \pm 4.34 \%$, similar to those of the parents (Table 2), indicating that the embryo sac of the $F_{1}$ hybrid was normal.

The distribution of pollen sterility in $200 \mathrm{HJX} 74 /$ SSSL-S23 $F_{2}$ plants showed a clear bimodal pattern with an apparent valley at $80-90 \%$ in NSD (Fig. 2e). Taking $80-90 \%$ as the cut-off region between sterility and fertility, the population segregated for sterility $(<80 \%)$ : fertility $(>90 \%)$ at the ratio of $1: 1\left(\chi^{2}=0.8<\chi_{0.05,1}^{2}=3.84\right)$, demonstrating monogenic inheritance for the trait. We concluded that hybrid male sterility in our population was determined by a single gene on the substituted segment of chromosome 7 (named as S23). Interestingly, the pollen sterility of $F_{1}$ hybrid could only be observed in NSD (Table 2 and Fig. 2c), but not in natural long-day (NLD) (Table 2 and Fig. 2d), suggesting the function of $S 23$ was environment-conditioned, and was possibly regulated by photoperiod and/or temperature.

The agronomic traits of HJX74, SSSL-S23 and their $\mathrm{F}_{1}$ hybrid were also evaluated in NSD (Fig. 3). No significant differences were found for plant height, panicle length, panicle exertion, number of tillers per plant, number of primary branches per panicle and seed setting rate among the three genotypes (Fig. 3). However, heading of $F_{1}$ hybrid was significantly delayed compared to the two parents, suggesting that $S 23$ might also have effect on heading date. The grain length, grain width and 100-grain weight were significantly lower in $F_{1}$ hybrid and SSSL-S23 compared with HJX74 (Fig. 3), suggesting that the substitution segment on

Table 2 Pollen and spikelet fertility of the parents and $F_{1}$ hybrid

\begin{tabular}{llll}
\hline Growth condition & Materials & Pollen fertility (\%) & Spikelet fertility (\%) \\
\hline NSD & HJX74 & $94.95 \pm 5.24 \mathrm{~A}^{\text {a }}$ & $92.63 \pm 4.31 \mathrm{~A}$ \\
& SSSL-S23 & $95.23 \pm 3.29 \mathrm{~A}$ & $90.21 \pm 4.98 \mathrm{~A}$ \\
& HJX74/SSSL- & $63.15 \pm 13.49 \mathrm{~B}$ & $89.61 \pm 4.34 \mathrm{~A}$ \\
& S23 & & \\
NLD & HJX74 & $93.91 \pm 1.94 \mathrm{~A}$ & $89.16 \pm 4.24 \mathrm{~A}$ \\
& SSSL-S23 & $94.48 \pm 2.14 \mathrm{~A}$ & $88.37 \pm 4.89 \mathrm{~A}$ \\
& HJX74/SSSL- & $92.99 \pm 2.38 \mathrm{~A}$ & $91.78 \pm 4.42 \mathrm{~A}$ \\
& S23 & & \\
\hline
\end{tabular}

Note: Pollen fertility and spikelet fertility were shown as mean \pm SD. ${ }^{a}$ Duncan's multiple comparison test was conducted for pollen fertility and spikelet fertility. Numbers followed by different letters in each row represented statistically significant difference at $P=0.01$, while numbers with the same letter indicated no significant difference at $P=0.05$ 


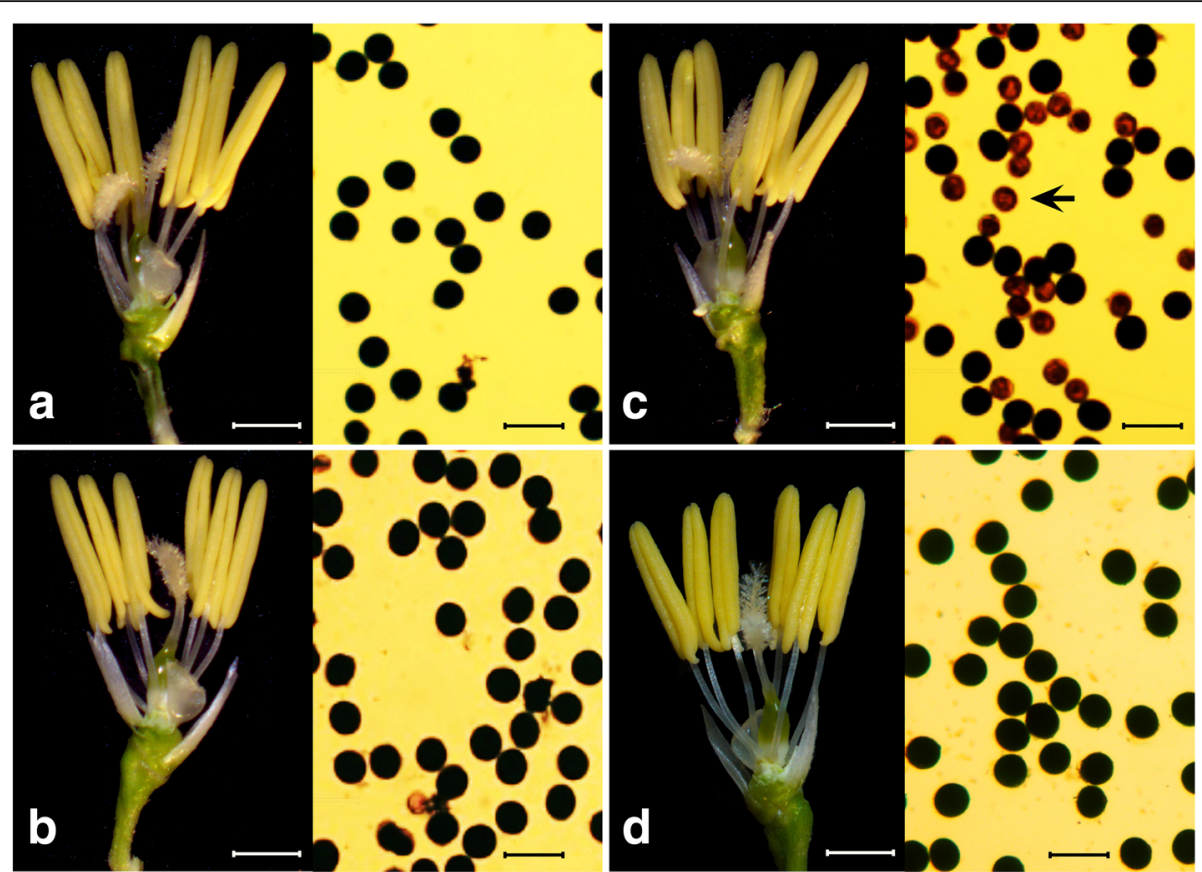

e

HJX74,

SSSL-S23

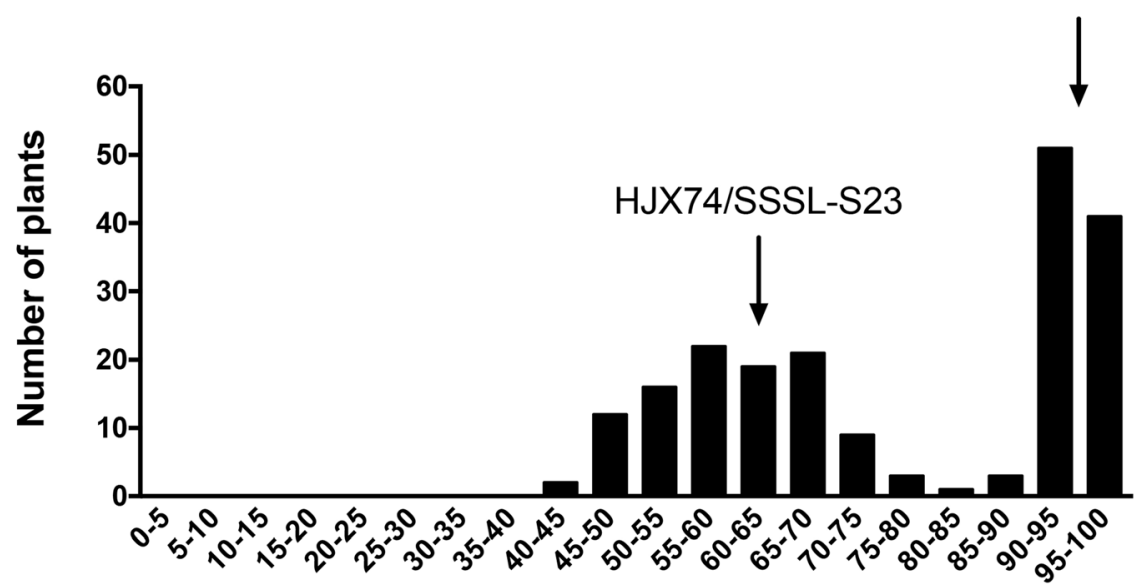

Pollen fertility (\%)

Fig. 2 Pollen fertility of HJX74, SSSL-S23, HJX74/SSSL-S23 $F_{1}$ and $F_{2}$ populations. Spikelets (left) and pollen grains (right) in HJX74 (a in NSD), SSSLS23 (b in NSD) and HJX74/SSSL-S23 F 1 (c in NSD and $\mathbf{d}$ in NLD) plants. Arrow indicated the aborted pollen grain. Scale bars, $1 \mathrm{~mm}$ for spikelet and $100 \mu \mathrm{m}$ for pollen. e A bimodal distribution for pollen fertility detected in $200 \mathrm{~F}_{2}$ plants derived from the cross between HJX74 and SSSL-S23 in NSD. Arrows indicated the mean pollen fertility of HJX74, SSSL-S23 and HJX74/SSSL-S23 F plants, respectively

chromosome 7 from wild rice might have a dominant QTL conferring small grain phenotype.

Fine mapping of $S 23$

Under NSD condition, two hundred HJX74/SSSL-S23 $\mathrm{F}_{2}$ plants were used to analyze the linkage between pollen fertility and SSR markers, and S23 locus was pinpointed to an interval between MM3659 and PSM147 (Fig. 4a). Twelve new markers were developed in the marker interval of MM3659-PSM147, and were used to analyze a total of $4500 \mathrm{~F}_{3}$ plants. As a result, nine recombinants were identified between markers ID6400 and SV-3 (Fig. 4b). The self-pollinated progenies $\left(\mathrm{F}_{4}\right.$ lines) of those nine plants were used to determine the genotypes of $S 23$ (Fig. 4c). Based on the sequence of a japonica standard, Nipponbare, the recombinants R-2 and R-3 restricted $S 23$ to $11.54 \mathrm{~kb}$ genomic region between the SNP-2 and SNP-3 markers, including three putative genes (Os07g0646500, Os07g0646600 and Os07g0646700, named as ORF4, ORF3 and ORF5, respectively) (Fig. 4c). 

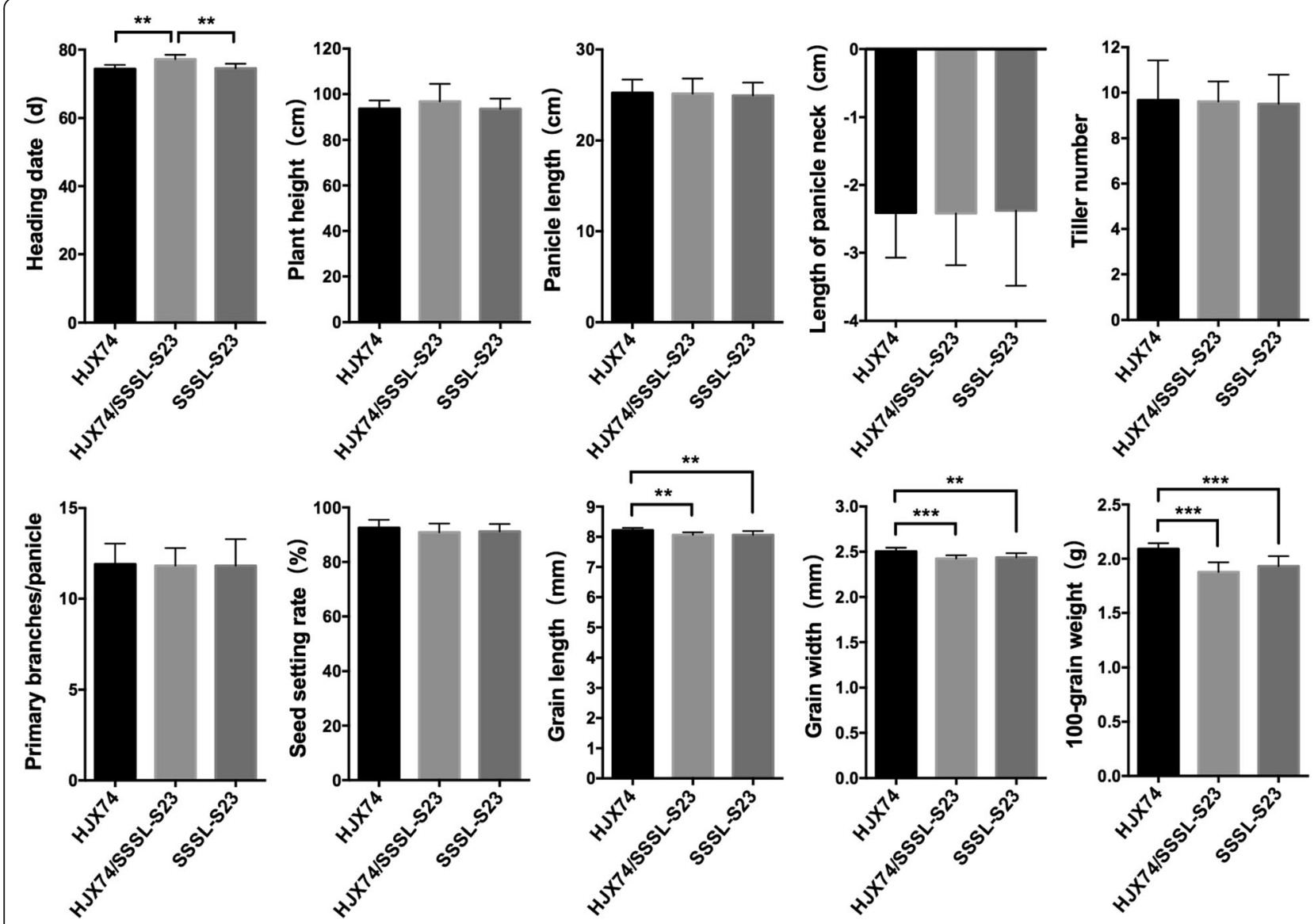

Fig. 3 Ten key agronomic traits in HJX74, SSSL-S23 and HJX74/SSSL-S23 F plants in NSD. ${ }^{* *}$ and ${ }^{* *}$ represented significance at $P=0.01$ and $P=0.001$, respectively

Sequence analysis revealed that there were also three genes $\left(O R F 3^{H J X 74}, O R F 4^{H J X 74}\right.$ and ORF5 $\left.5^{H J X 74}\right)$ in the $11.54 \mathrm{~kb} \mathrm{S23}$ region in HJX74, but only two genes (ORF4Glu and ORF5 ${ }^{\text {Glu }}$ ) in the $7.08 \mathrm{~kb} S 23$ region in SSSL-S23 (Fig. 4d). Both ORF4 and ORF5 are predicted to encode a SWIM-type zinc finger and MULE transposase domaincontaining protein, and ORF3 is predicted to encode a 496-aa protein without any conserved domains. Sequence comparation of the candidate genes in HJX74 and SSSL-S23 revealed nucleotide diversity for ORF5 but not for ORF4. Four SNPs were found in the coding region of ORF5 between HJX74 and SSSL-S23, resulting in two amino acid substitutions (Additional file 1: Figure S2). Interestingly, a 288-bp deletion was found in ORF5 ${ }^{H J X 74}$ promoter region compared to that of ORF5 ${ }^{\text {Glu }}$ (Fig. 4d). Indel (Insertion and deletion) was also found in Os07g0646400 (named as ORF2) which located just outside of the candidate mapping region of $S 23$. Compared to the sequence of $O R F 2^{H J X 74}$, a 398-bp insertion, a 44-bp deletion and several SNPs were found in $O R F 2^{G l u}$, resulting in sequence difference of a 24-residue insertion/ deletion and eight amino acid substitutions for the encoded proteins (Fig. 4d and Additional file 1: Figure S3a).

\section{Candidate gene analysis of $\mathbf{S 2 3}$}

To define candidate genes for $S 23$, we examined the expression of the three target genes as well as ORF2 in which major sequence differences were found between the two parent genotypes in NSD. All genes were highly expressed in different stages of anther development, but only ORF3 and ORF2 showed expression in vegetative organs (Fig. 5). ORF4 was equally expressed among HJX74, SSSL-S23 and $F_{1}$ hybrid (Fig. 5a). In combination with the consideration that ORF4 sequence was identical between HJX74 and SSSL-S23, we speculated that ORF4 was not the candidate gene for S23. ORF3 was stably expressed during anther development, while ORF5 expression was slightly higher in early stages of anther development, and was gradually reduced in later stages (Fig. 5b and c). As expected, no expression of ORF3 was detected in SSSL-S23 since ORF3 was absent in SSSL-S23 (Fig. 5b). Interestingly, ORF5 was expressed in 


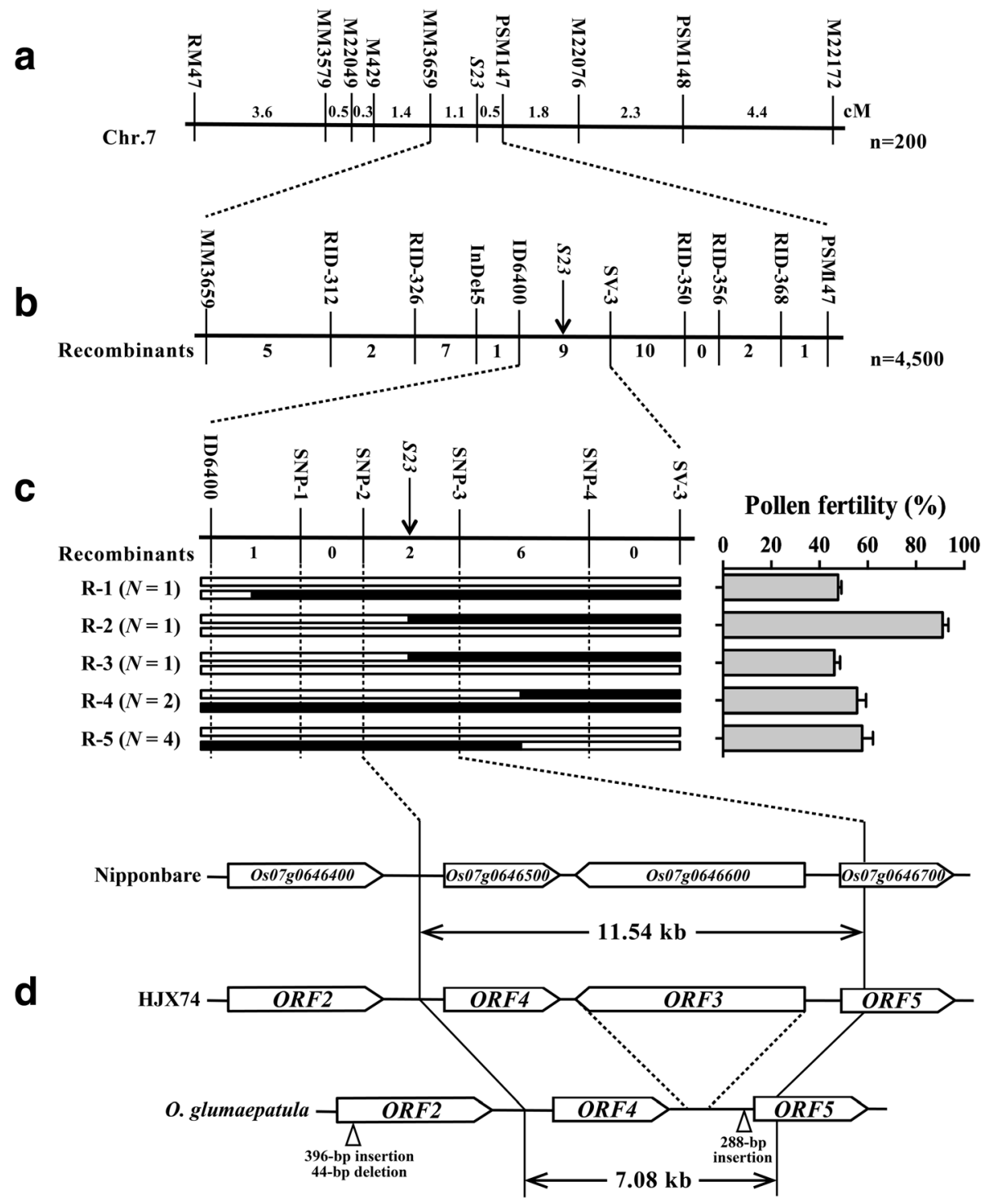

Fig. 4 Map-based cloning of S23. (a and b) S23 was mapped to a 1.6 CM region flanked by MM3659 and PSM147 using 200 HJX74/SSSL-S23 F 2 plants (a), and was then fine-mapped to the region between ID6400 and SV-3 using 4500 HJX74/SSSL-S23 F 3 plants (b). c Nine key recombinants defined S23 to the region between SNP-2 and SNP-3. In Nipponbare genome, there were three genes in the $11.54 \mathrm{~kb}$ target region between SNP-2 and SNP-3. White and black boxes indicated chromosomal segments from HJX74 and O. glumaepatula, respectively. Genetic distance and the number of recombinants between adjacent markers was shown above and below the bar, respectively. $\mathbf{d}$ Sequence analysis was performed for the fine-mapped region and neighbouring region of HJX74 and O. glumaepatula, and ORFs were predicted

SSSL-S23, but not in HJX74 (Fig. 5c), suggesting that the 288-bp deletion in HJX74 completely disrupted the expression of ORF5. In $\mathrm{F}_{1}$ hybrid, the expression of ORF3 and ORF5 was about half of that in HJX74 and SSSL-S23, respectively (Fig. 5b and c), in consistent with that only one allele of ORF3 and ORF5 was expressed in $F_{1}$ hybrid. The expression of ORF2 was lower in early stages of anther development, and was gradually increased in later stages (Fig. 5d). Furthermore, ORF2 was equally expressed among HJX74, SSSL-S23 and $F_{1}$ hybrid (Fig. 5d), suggesting the major sequence differences identified in ORF2 possibly interfered with the protein function instead of affecting its transcription.

\section{Discussion}

The wild species of Oryza are an important reservoir of useful genes. Numerous genes conferring resistance or tolerance to biotic and abiotic stresses have been identified in wild species of Oryza (Brar and Khush 1997). Although the overall economical characters of wild rice 

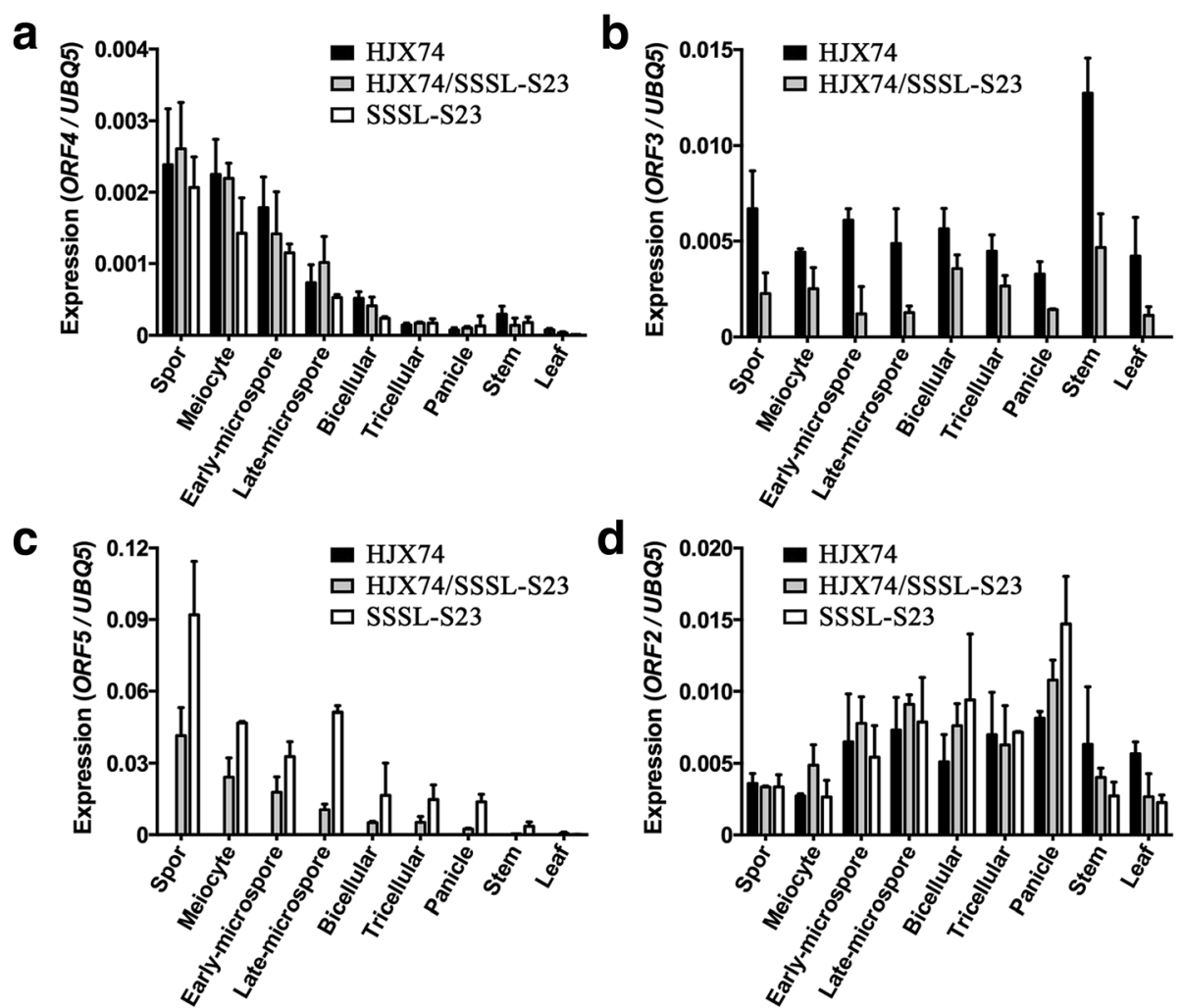

Fig. 5 Expression analysis of $S 23$ candidate genes. The expression of ORF4 (a), ORF3 (b), ORF5 (c) and ORF2 (d) was analyzed in developing anthers from sporogenous cell stage to tricellular pollen stage and in other tissues including panicles, stems and leaves of HJX74, SSSL-S23 and HJX74/SSSL-S23 $F_{1}$ plants in NSD. Spor, sporogenous cell stage

are inferior to those of cultivated rice, previous reports demonstrated that many favorable genes hidden in wild rice are essential for yield-related trait improvement (Xiao et al. 1996, 1998; Tian et al. 2006; Huang et al. 2012). However, hybrid sterility, which is the most common form of postzygotic isolation and plays an important role in maintaining species identity (Orr and Presgraves 2000), is the major obstacle for the utilization of $O$. glumaepatula specific traits in improving rice varieties. Until now, only a few pollen eliminators have been identified in the hybrid between $O$. sativa and O. glumaepatula, and only S27/S28 gene pairs had been molecularly analyzed (Sano 1994; Sobrizal et al. 2000a, b, Sobrizal and Yoshimura, 2001, 2002; Yamagata et al. 2010; Sakata et al. 2014; Zhang et al. 2018). In order to identify and transfer favorable alleles from O. glumaepatula into cultivated rice varieties, and to facilitate their application in rice breeding, a set of SSSLs was developed in the genetic background of an elite indica cultivar HJX74 using wild rice O. glumaepatula as the donor parent. During the process of SSSLs development, an interspecific hybrid sterile locus $S 23$ was identified to be involved in male gamete development in hybrids between O. sativa and O. glumaepatula, and was then fine mapped to $11.54 \mathrm{~kb}$ and $7.08 \mathrm{~kb}$ genomic region in $O$. sativa and O. glumaepatula, respectively, encoding three and two candidate ORFs, respectively. $S 23$ was first identified in 2000 during the development of O. glumaepatula introgression lines in the background of $O$. sativa $\mathrm{cv}$. Taichuang 65, and it was found to be co-segregated with RFLP marker C1340 (Sobrizal et al. 2000a), which was close to the position of $S 21$ from O. glaberrima and O. rufipogon for hybrid male sterility (Doi et al. 1999; Miyazaki et al. 2006). Recently, $q H M S 7$, which conferred hybrid male sterility between $O$. meridionalis and $O$. sativa, was cloned and contained two tightly linked genes (ORF2 and ORF3) (Yu et al. 2018). ORF2, which was functional in $O$. sativa but was non-functional in $O$. meridionalis, encoded a toxin affecting the development of pollen, and ORF3, which was present in $O$. sativa but was missing in $O$. meridionalis, encoded an antidote required for pollen viability. Mer-type pollens lacking ORF3 were selectively eliminated, leading to segregation distortion in the progeny. Sequence analysis revealed that $S 23$ was allelic with $q H M S 7$, and S23-ORF3 was identical to qHMS7-ORF3 (Additional file 1: Figure S3b). Interestingly, $q H M S 7-O R F 2$ didn't locate in the mapping region of $S 23$, since the recombinants R-2 
and R-3 restricted $S 23$ to the genomic region between the SNP-2 and SNP-3 markers (Fig. 4c). However, sequencing of the recombinants $\mathrm{R}-2$ and $\mathrm{R}-3$ revealed that both recombinants fitted the "Toxin-Antidote" model of $q H M S 7$ as they both contained ORF2 allele from O. sativa, while one and two alleles of ORF3 existed in R-3 and R-2, respectively (Additional file 1: Figure S4). Therefore, when map-based cloning QTLs of hybrid sterility, neighboring regions of the target site should be also analyzed even if the result of the recombinants has suggested otherwise.

Although S23 was allelic to $q H M S 7$, the genetic effect was not completely the same. $F_{1}$ pollen fertility was $51.16 \pm 1.29 \%$ for $q H M S 7$. Gametophytic transmission analysis using the backcross population of NIL-qHMS7/DJY1 and the self-pollinated population of NIL-qHMS7 identified no heterozygous genotype and no homozygote from O. meridionalis at qHMS7 locus, respectively, indicating the pollen grains carrying O. meridionalis allele aborted completely ( $\mathrm{Yu}$ et al. 2018). However, $F_{1}$ pollen fertility was $63.15 \pm 13.49 \%$ for S23 (Table 2), and homozygote from O. glumaepatula at S23 locus could be identified (Table 1), indicating that the pollen grains carrying O. glumaepatula allele was not completely sterile. We speculated that the "Toxin" in our study was not as toxic as that in $q H M S 7$ research, or the "Antidote" in S23 locus was super effective. Since ORF2 was equally expressed among HJX74, SSSL-S23 and F F $_{1}$ hybrid (Fig. $5 \mathrm{~d}$ ), a possible explanation of the phenotypic difference might be the different ORF2 function due to sequence variation. Although $q H M S 7-O R F 2$ was sequenced in 89 accessions of wild Oryza species, 210 accessions of $O$. sativa and 11 accessions of O. glaberrima collected from 29 countries and regions over diverse geographical area, and 27 haplotypes were identified among the sequenced accessions, ORF2 sequence in O. glumaepatula was untouched (Yu et al. 2018). Our sequence analysis revealed a 24-residue insertion/deletion and eight amino acid substitutions between $\mathrm{ORF}^{\mathrm{Glu}}$ and ORF2 ${ }^{\mathrm{HIX74}}$ (Additional file 1: Figure S3a). However, the sequence of functional S23-ORF2 $2^{\mathrm{HJX} 74}$ and qHMS7-ORF2 $^{\mathrm{D}}$ was identical (Additional file 1: Figure S3a), indicating the toxin function of S23-ORF2 ${ }^{\mathrm{HIX} 74}$ was not affected compared with qHMS7-ORF2 ${ }^{\mathrm{D}}$. Furthermore, ORF3 sequence was completely same between $\mathrm{ORF}^{\mathrm{D}}$ in $q H M S 7$ and $\mathrm{ORF}^{\mathrm{HIX} 74}$ in $S 23$ (Additional file 1: Figure S3b), and the expression of $O R F 3^{H J X 74}$ was unaffected (Fig. 5b), indicating that the efficacy of the antidote S23-ORF3 ${ }^{\mathrm{HIX} 74}$ might not be the reason for the different genetic effect. Therefore, an unidentified partner should play some different role in S23 and qHMS7. A possible candidate was ORF1, which was homologous to ORF3 and located just next to ORF2 (Yu et al. 2018). Several polymorphic sites near the C-terminal region of ORF1 compared with ORF3 might affect the protein function (Additional file 1: Figure S3b). Furthermore, transformation of intact $O R F 1^{D}$ genomic sequence into the hybrid D/M-type plants could not restore the pollen fertility, suggesting that $\mathrm{ORF} 1^{\mathrm{D}}$ was non-functional ( $\mathrm{Yu}$ et al. 2018). However, the expression of ORF1 ${ }^{H J X 74}$, which was supposed to similar to the $O$. sativa allele $O R F 1^{D}$, was nearly undetectable, while the expression of $O R F 1^{\text {Glu }}$ reached about onetenth to one-fifth expression level of ORF3 (Fig. 5b and Additional file 1: Figure S5). Therefore, trace level of $O R F 1^{G l u}$ could possibly partially restored the pollen fertility in HJX74/SSSL-S23 $\mathrm{F}_{1}$ hybrid plants. ORF5 could also be a possible candidate. A 288-bp deletion were found in $O R F 5^{H J X 74}$ promoter region compared to that of $O R F 5^{\text {Glu }}$ (Fig. 4d), and completely disrupted the expression of ORF5 ${ }^{H J X 74}$. Whether ORF5 ${ }^{\text {Glu }}$ performs an antidote-like function to alleviate the toxicity of $\mathrm{ORF} 2^{\mathrm{HJX} 74}$ remains to be investigated.

Another phenotypic difference caused by $S 23$ and qHMS7 was that the pollen sterility of $\mathrm{F}_{1}$ hybrid in our study could only be observed in NSD, but not in NLD (Table 2, Fig. 2c and d). Fertile pollen in $F_{1}$ hybrid of HJX74 and SSSL-S23 had been observed in the NLD of four consecutive years in Guangzhou, China, suggesting the function of $S 23$ was environment-conditioned. Since $\mathrm{ORF}^{\mathrm{HIX74}}$ in $S 23$ was identical to ORF2 ${ }^{\mathrm{D}}$ in $q H M S 7$, we assumed that the expression of ORF2 $2^{H J X 74}$ might be regulated by photoperiod and/or temperature, resulting in the absence or low level of toxin in NLD. The trait of photoperiod- and/or thermo-sensitive male fertility-sterility conversion had been widely used in two-line system in hybrid rice breeding, and the underlying mechanisms of some acting genes and lncRNAs had been recently studied (Zhou et al. 2012, 2014; Ding et al. 2012; Zhang et al. 2013; Fan et al. 2016; Fan and Zhang 2018). However, none of them had been reported to be involved in hybrid sterility. To our knowledge, this is the first report on a single locus controlling both hybrid sterility and environment-sensitive sterility. Thus, our findings provided a valuable example of $S 23$ to study the crosstalk between $F_{1}$ hybrid male sterility and environment-conditioned male sterility in rice.

S23 causing male gamete abortion in the heterozygous condition limits the utilization of favorable genes not only in O. glumaepatula, but also in other wild Oryza species. Understanding the nature of this gene offers approaches to overcome male sterility in wild rice-cultivated rice hybrids, thus facilitating utilization of the strong hybrid vigor. $O$. sativa carrying non-functional S23-ORF2 can be selected as wide-compatible lines to break down the reproductive barrier and overcome hybrid sterility in the interspecific hybrid breeding. Furthermore, the genome editing-based approach can rapidly generate neutral alleles at $S 23$ by creating 
loss-of-function S23-ORF2, allowing broader and easier access to desirable traits in distantly related species.

\section{Conclusions}

A set of SSSLs was developed in the genetic background of an elite indica cultivar HJX74 using wild rice O. glumaepatula as the donor parent, and S23 was identified to cause both interspecific hybrid male sterility and environment-conditioned male sterility in rice. Identification and candidate genes analysis of S23 in this study provides a valuable example to study the crosstalk between interspecific $F_{1}$ hybrid male sterility and environment-conditioned male sterility in rice, facilitates reserving and utilizing favorable genes or alleles of wild Oryza species, and allows for a more efficient exploitation of distant heterosis in hybrid rice breeding.

\section{Methods}

\section{Plant materials and growth conditions}

To develop SSSLs, the recipient parent HJX74, an elite indica variety from south China, was crossed with the donor parent wild rice O. glumaepatula (accession number IRGC104387), and the $F_{1}$ plants were backcrossed with HJX74 to develop the $\mathrm{BC}_{1} \mathrm{~F}_{1}$ generation. Polymorphic SSR markers were used in the selection of the donor chromosomal segments. Using the same method, $\mathrm{BC}_{5} \mathrm{~F}_{1}$ plants were obtained, and were self-crossed to produce $\mathrm{BC}_{5} \mathrm{~F}_{2}$ lines in which the majority of genomic regions were homozygous for HJX74 alleles. 191 polymorphic markers evenly distributed over 12 chromosomes were selected to detect the chromosome substituted segments. SSSL which had only one substituted segment from the wild rice O. glumaepatula could be obtained in this generation. Plants with two or more substituted segments would be crossed with HJX74 again to produce more SSSLs.

Plants were grown under NSD (from July to November) and NLD (from March to July) conditions in a paddy field in Guangzhou $\left(23^{\circ} 07^{\prime} \mathrm{N}, 113^{\circ} 15^{\prime} \mathrm{E}\right)$, China. In NSD, the average temperature in July, August, September, October and November is approximately $27.9^{\circ} \mathrm{C}, 28.7^{\circ} \mathrm{C}, 27.9^{\circ} \mathrm{C}$, $23.6^{\circ} \mathrm{C}$ and $19.3^{\circ} \mathrm{C}$, respectively, while in $\mathrm{NLD}$, the average temperature in March, April, May, June and July is approximately $17.9^{\circ} \mathrm{C}, 21.6^{\circ} \mathrm{C}, 25.1{ }^{\circ} \mathrm{C}, 28.2^{\circ} \mathrm{C}$ and $27.9^{\circ} \mathrm{C}$, respectively.

\section{PCR analysis and development of molecular markers}

Fresh leaves were collected at the seedling stage and then ground in liquid nitrogen. Microquantities of DNA were extracted from fresh leaves of each individual using a previously reported method (Chen et al. 2014). Amplification was carried out on the program for the initial denaturing step with $94{ }^{\circ} \mathrm{C}$ for $3 \mathrm{~min}$, followed by 35 cycles for $30 \mathrm{~s}$ at $94{ }^{\circ} \mathrm{C}, 30 \mathrm{~s}$ at $55^{\circ} \mathrm{C}, 30 \mathrm{~s}$ at $72{ }^{\circ} \mathrm{C}$, with a final extension at $72^{\circ} \mathrm{C}$ for $5 \mathrm{~min}$. PCR products were separated on $6 \%$ nondenaturing polyacrylamide gel and detected using the silver staining method. SSR markers were selected covering the target region based on the published linkage map of rice (http://www.gramene.org). Indel and SNP markers used for fine mapping of $S 23$ were designed based on the result of the sequence analysis of HJX74 and SSSL-S23.

\section{Examination of pollen and spikelet fertility}

The examination of pollen and spikelet fertility was as described previously (Guo et al. 2016). Briefly, to examine pollen fertility, 6-9 mature flowers were collected from the upper one-third of the panicles of plants during the flowering time and fixed in FAA solution (ethanol, formaldehyde and acetic acid at a ratio of 89:6:5). The pollen was stained with $1 \% \mathrm{I}_{2}$-KI solution containing $0.1 \%(w / v)$ iodine and $1 \%(\mathrm{w} / \mathrm{v})$ potassium iodide. More than 300 pollen grains were randomly scanned per plant. The pollen could be divided into two types: normal pollen (normal size and fully stained) and stained abortive pollen (small size and lightly stained). Pollen fertility was estimated by the proportion of fertile pollen grains present. To examine spikelet fertility, three main panicles per plant were harvested and calculated for the mean seed set. Ten to twenty plants were recorded for each variation.

\section{Agronomic traits evaluation}

Agronomic traits were evaluated under NSD condition. Heading date was defined as the time when the first panicle emerged. Plant height and number of tillers per plant was measured at maturity stage and tilling stage, respectively. Yield-related traits including panicle length, panicle exertion, number of primary branches per panicle, seed-setting rate, grain length, grain width and 100-grain weight were investigated after rice harvested at maturity stage and sun dried. Panicle exertion was calculated as the distance between the leaf cushion of flag and the neck-panicle node. Grain length and grain width were determined by using Microtek ScanWizard EZ scanner and SC-E Image analysis software (Hangzhou Wanshen Detection Technology Co., Ltd., Hangzhou, China). Ten to twenty plants were recorded for each variation. Differences among the three genotypes were determined using one-way ANOVA.

\section{Sequence analysis}

A number of primer pairs were designed based on the reference Nipponbare genomic sequence and longlength PCR with KOD Plus Neo DNA polymerase (TOYOBO, Japan) was used to amplify the $S 23$ region sequences from gDNAs of HJX74 and SSSL-S23 in 
multiple fragments. The PCR products were then sequenced. The sequences were assembled using SeqMan of the Lasergene package, and analyzed by BLAST in VectorNTI package.

\section{RNA isolation, RACE and qRT-PCR analysis}

Total RNAs from rice tissues (anthers, young panicles, leaves and stems) of HJX74, SSSL-S23 and their hybrid were isolated using TRIZOL reagent (Invitrogen) following the manufacturer's instruction. First-strand cDNA was reverse transcribed from DNaseI-treated RNA with oligo-dT as the primer using ReverTra Ace kit (Toyobo). For the RACE assay, the full-length transcripts were amplified by nested PCR with the SMARTer RACE cDNA Amplification kit (Clontech). Gene expression was measured by qRT-PCR using the ABI 7500 system (Life technologies). The qRT-PCR was carried out in a total volume of $20 \mu \mathrm{l}$ containing $1 \times$ SYBR Green Master Mix (Life technologies). We normalized the expression levels by using UBQ 5 gene as internal control. Each set of experiments was repeated three times, and the relative standard curve quantification method was used to evaluate quantitative variation. The qRT-PCR procedure was conducted at $94^{\circ} \mathrm{C}$ for $3 \mathrm{~min}$, followed by 40 cycles at $94{ }^{\circ} \mathrm{C}$ for $30 \mathrm{~s}, 58^{\circ} \mathrm{C}$ for $30 \mathrm{~s}$, and $72^{\circ} \mathrm{C}$ for $30 \mathrm{~s}$. The primers used were listed in Additional file 2: Table S1.

\section{Additional files}

Additional file 1: Figure S1. Graphic genotype of NIL7. Black bars indicated the genomic fragments from O. glumaepatula and the other parts were from HJX74. Figure S2. Alignment of the deduced amino acid sequence of ORF5 ${ }^{\text {Glu }}$ and ORF $5^{\text {HJX74 }}$. The two amino acid substitutions were shown in red. Figure S3. Haplotype analysis of ORF2 and ORF1/3. The gene structures of ORF2 (a) and ORF1/ORF3 (b) were shown on the top and on the bottom, respectively. The black and grey blocks indicated translated regions and untranslated regions, respectively. The variable sites were shown by the vertical lines. The site numbers indicated the positions of amino acid in ORF2 ${ }^{\mathrm{HJX} 74} / \mathrm{ORF}^{\mathrm{D}}$ and ORF3 for the respective polymorphic sites, respectively. The haplotype numbers in parentheses indicated the length of the deduced proteins for the respective haplotypes. ORF $2^{\mathrm{HJ} X 74} / \mathrm{ORF} 2^{\mathrm{D}}$ and ORF3 were functional, while ORF2 ${ }^{\text {Glu }}$, ORF2 ${ }^{\mathrm{M}}$ and different haplotypes of ORF1 were supposed to be non-functional. The polymorphic sites in ORF2 ${ }^{\text {Glu }}$ compared with ORF2 ${ }^{\mathrm{M}}$, in ORF1 ${ }^{\mathrm{HJX} X 4}$ and in $\mathrm{ORF} 1^{\mathrm{M}}$ compared with ORF1 ${ }^{\mathrm{D}} / \mathrm{ORF}^{\text {Glu }}$ were labelled in grey. Figure S4. Graphic genotype of the two key recombinants R-2 and $\mathrm{R}-3$. White and black boxes indicated chromosomal segments from HJX74 and O. glumaepatula, respectively. Black triangle represented 288-bp insertion in ORF5 $5^{G / u}$ promoter region compared to that of ORF5 $5^{H X 74}$. Figure S5. Expression analysis of S23-ORF1. The expression of ORF1 was analyzed in developing anthers from sporogenous cell stage to tricellular pollen stage and in other tissues including panicles, stems and leaves of HJX74, SSSL-S23 and HJX74/SSSL-S23 F 1 plants in NSD. Spor, sporogenous cell stage. (PPT $1720 \mathrm{~kb}$ )

Additional file 2: Table S1. Primers used in this study. (DOCX 84 kb)

\section{Abbreviations}

Indel: Insertion and deletion; NLD: Natural long-day; NSD: Natural short-day; ORF: Open reading frame; SNP: Single nucleotide polymorphism; SSR: Simple sequence repeat; SSSLs: Chromosome single-segment substitution lines
Acknowledgments

Not applicable.

\section{Funding}

This work was supported by National Natural Science Foundation of China (31571483 and 31671762), Guangdong Provincial Natural Science Foundation (2015A030313415), Presidential Fund of South China Agriculture University (4100-K12225), and Guangzhou Scientific and Technological Plan (201804020086)

\section{Availability of data and materials}

All data and materials used and/or analyzed during the current study are available from the corresponding author on request.

\section{Authors' contributions}

ZL conceived and designed the experiment. CF and LL conducted the experiments, performed data analysis. $\mathrm{RH}, \mathrm{DW}, \mathrm{MW}, \mathrm{QH}, \mathrm{QM}, \mathrm{KQ}, \mathrm{XYF}, \mathrm{XLF}$ and GZ participated in material development, molecular marker analysis and agronomic traits evaluation. ZL drafted the proposal and wrote the manuscript. All authors read and approved the final manuscript.

Ethics approval and consent to participate

Not applicable.

\section{Consent for publication}

Not applicable.

\section{Competing interests}

The authors declare that they have no competing interests.

\section{Publisher's Note}

Springer Nature remains neutral with regard to jurisdictional claims in published maps and institutional affiliations.

\section{Author details}

${ }^{1}$ State Key Laboratory for Conservation and Utilization of Subtropical Agro-Bioresources, South China Agricultural University, Guangzhou 510642, China. ${ }^{2}$ Guangdong Provincial Key Laboratory of Plant Molecular Breeding, College of Agriculture, South China Agricultural University, Guangzhou 510642, China. ${ }^{3}$ Present address: Huazhi Rice Bio-Tech Co., Ltd., Changsha 410124, China. ${ }^{4}$ Present address: Vegetable Research Institute, Guangdong Academy of Agricultural Sciences, Guangzhou 510642, China.

Received: 10 September 2018 Accepted: 18 February 2019

Published online: 28 February 2019

\section{References}

Brar DS, Khush GS (1997) Alien Introgression in Rice. Plant Mol Biol 35:35-47. https://doi.org/10.1023/A:1005825519998

Chen J, Ding J, Ouyang Y et al (2008) A triallelic system of S5 is a major regulator of the reproductive barrier and compatibility of indica-japonica hybrids in rice. Proc Natl Acad Sci 105:11436-11441. https://doi.org/10.1073/pnas. 0804761105

Chen J, Li X, Cheng C et al (2014) Characterization of epistatic interaction of QTLS LH8 and EH3 controlling heading date in Rice. Sci Rep 4:4263. https://doi.org/ $10.1038 /$ srep04263

Ding J, Shen J, Mao H et al (2012) RNA-directed DNA methylation is involved in regulating photoperiod-sensitive male sterility in Rice. Mol Plant 5:1210-1216. https://doi.org/10.1093/mp/sss095

Doi K, Taguchi K, Yoshimura A (1999) RFLP mapping of S20 and S21 for F 1 pollen semi-sterility found in backcross progeny of Oryza sativa and O. glaberrima. Rice Genet Newsl 16:65-67

Fan Y, Yang J, Mathioni SM et al (2016) PMS1T, producing phased smallinterfering RNAs, regulates photoperiod-sensitive male sterility in rice. Proc Natl Acad Sci 113:15144-15149. https://doi.org/10.1073/pnas.1619159114

Fan Y, Zhang Q (2018) Genetic and molecular characterization of photoperiod and thermo-sensitive male sterility in rice. Plant Reprod 31:3-14. https://doi. org/10.1007/s00497-017-0310-5

Guo J, Xu X, Li W et al (2016) Overcoming inter-subspecific hybrid sterility in rice by developing indica-compatible japonica lines. Sci Rep 6:26878. https://doi. org/10.1038/srep26878 
He N, Wu R, Pan X et al (2017) Development and trait evaluation of chromosome single-segment substitution lines of $\mathrm{O}$. meridionalis in the background of $\mathrm{O}$. sativa. Euphytica 213:281. https://doi.org/10.1007/s10681-017-2072-4

Huang X, Zhao Y, Wei X et al (2012) Genome-wide association study of flowering time and grain yield traits in a worldwide collection of rice germplasm. Nat Genet 44:32-39. https://doi.org/10.1038/ng.1018

Koide Y, Ogino A, Yoshikawa T et al (2018) Lineage-specific gene acquisition or loss is involved in interspecific hybrid sterility in rice. Proc Natl Acad Sci 115: E1955-E1962. https://doi.org/10.1073/pnas.1711656115

Kubo T, Takashi T, Ashikari M et al (2016) Two tightly linked genes at the hsal locus cause both $F_{1}$ and $F_{2}$ hybrid sterility in Rice. Mol Plant 9:221-232. https://doi.org/10.1016/j.molp.2015.09.014

Long Y, Zhao L, Niu B et al (2008) Hybrid male sterility in rice controlled by interaction between divergent alleles of two adjacent genes. Proc Natl Acad Sci 105:18871-18876. https://doi.org/10.1073/pnas.0810108105

McCouch SR, Sweeney M, Li J et al (2007) Through the genetic bottleneck: O. rufipogon as a source of trait-enhancing alleles for O. sativa. Euphytica 154: 317-339. https://doi.org/10.1007/s10681-006-9210-8

Miyazaki Y, Doi K, Yasui $H$, Yoshimura A (2006) Identification of a new allele of $F_{1}$ pollen sterility gene, $\mathbf{S 2 1}$, detected from the hybrid between Oryza sativa and O. rufipogon. Rice Genet Newsl 23:36-38

Mizuta Y, Harushima Y, Kurata N (2010) Rice pollen hybrid incompatibility caused by reciprocal gene loss of duplicated genes. Proc Natl Acad Sci 107:20417-20422. https://doi.org/10.1073/pnas.1003124107

Nguyen GN, Yamagata Y, Shigematsu Y et al (2017) Duplication and loss of function of genes encoding RNA polymerase III subunit C4 causes hybrid incompatibility in Rice. G3 (Bethesda) 7:2565-2575. https://doi.org/10.1534/ g3.117.043943

Orr HA, Presgraves DC (2000) Speciation by postzygotic isolation: forces, genes and molecules. BioEssays 22:1085-1094. https://doi.org/10.1002/1521-1878

Ouyang Y, Chen J, Ding J, Zhang Q (2009) Advances in the understanding of inter-subspecific hybrid sterility and wide-compatibility in rice. Chin Sci Bull 54:2332-2341. https://doi.org/10.1007/s11434-009-0371-4

Ouyang Y, Zhang Q (2013) Understanding reproductive isolation based on the Rice model. Annu Rev Plant Biol 64:111-135. https://doi.org/10.1146/annurevarplant-050312-120205

Sakata M, Yamagata Y, Doi K, Yoshimura A (2014) Two linked genes on rice chromosome 2 for $F_{1}$ pollen sterility in a hybrid between Oryza sativa and $O$. glumaepatula. Breed Sci 64:309-320. https://doi.org/10.1270/jsbbs.64.309

Sano Y (1994) Pollen-killer in rice. Japan J Breed 44:298

Shen R, Wang L, Liu X et al (2017) Genomic structural variation-mediated allelic suppression causes hybrid male sterility in rice. Nat Commun 8:1310. https:// doi.org/10.1038/s41467-017-01400-y

Sobrizal MY, Sanchez PL et al (2000a) Mapping of $F_{1}$ pollen semi-sterility gene found in backcross progeny of Oryza sativa L. and Oryza glumaepatula Steud. Rice Genet Newsl 17:61-63

Sobrizal MY, Sanchez PL et al (2000b) Identification of a gene for male gamete abortion in backcross progeny of Oryza sativa and Oryza glumaepatula. Rice Genet News| 17:59-61

Sobrizal MY, Yoshimura A (2001) Mapping of a gene for pollen semi-sterility on chromosome 8 of rice. Rice Genet Newsl 18:59-61

Sobrizal MY, Yoshimura A (2002) Mapping of pollen semi-sterility gene, S28(t), on rice chromosome 4. Rice Genet Newsl 19:80-82

Tanksley SD, McCouch SR (1997) Seed banks and molecular maps: unlocking genetic potential from the wild. Science 277:1063-1066. https://doi.org/10. 1126/science.277.5329.1063

Tian F, Li DJ, Fu Q et al (2006) Construction of introgression lines carrying wild rice (Oryza rufipogon Griff.) segments in cultivated rice (Oryza sativa L.) background and characterization of introgressed segments associated with yield-related traits. Theor Appl Genet 112:570-580. https://doi.org/10.1007/ s00122-005-0165-2

Xiao J, Grandillo S, Ahn SN et al (1996) Genes from wild rice improve yield. Nature 384:223-224. https://doi.org/10.1038/384223a0

Xiao J, Li J, Grandillo S et al (1998) Identification of trait-improving quantitative trait loci alleles from a wild rice relative, Oryza rufipogon. Genetics 150:899909. https://doi.org/10.1016/j.tplants.2012.11.001

Xie Y, Xu P, Huang J et al (2017) Interspecific hybrid sterility in Rice is mediated by OgTPR1 at the $S 1$ locus encoding a peptidase-like protein. Mol Plant 10: 1137-1140. https://doi.org/10.1016/j.molp.2017.05.005
Yamagata Y, Yamamoto E, Aya K et al (2010) Mitochondrial gene in the nuclear genome induces reproductive barrier in rice. Proc Natl Acad Sci 107:1494-1499. https://doi.org/10.1073/pnas.0908283107

Yang J, Zhao X, Cheng K et al (2012) A killer-protector system regulates both hybrid sterility and segregation distortion in Rice. Science 337:1336-1340. https://doi.org/10.1126/science.1223702

Yu X, Zhao Z, Zheng X et al (2018) A selfish genetic element confers nonMendelian inheritance in rice. Science 360:1130-1132. https://doi.org/10. 1126/science.aar4279

Yu Y, Zhao Z, Shi Y et al (2016) Hybrid sterility in Rice (Oryza sativa L.) involves the Tetratricopeptide repeat domain containing protein. Genetics 203:1439-1451. https://doi.org/10.1534/genetics.115.183848

Zhang H, Xu C, He Y et al (2013) Mutation in CSA creates a new photoperiodsensitive genic male sterile line applicable for hybrid rice seed production. Proc Natl Acad Sci 110:76-81. https://doi.org/10.1073/pnas.1213041110

Zhang Y, Zhou J, Li J et al (2018) Mapping of S56(t) responsible for interspecific hybrid sterility between Oryza sativa and Oryza glumaepatula. Breed Sci 68: 242-247. https://doi.org/10.1270/jsbbs.17116

Zhou H, Liu Q, Li J et al (2012) Photoperiod- and thermo-sensitive genic male sterility in rice are caused by a point mutation in a novel noncoding RNA that produces a small RNA. Cell Res 22:649-660. https://doi.org/10. $1038 / \mathrm{cr} .2012 .28$

Zhou H, Zhou M, Yang Y et al (2014) RNase $Z^{S 1}$ processes U $b_{L 40}$ mRNAs and controls thermosensitive genic male sterility in rice. Nat Commun 5:4884 https://doi.org/10.1038/ncomms5884

\section{Submit your manuscript to a SpringerOpen ${ }^{\circ}$ journal and benefit from:}

- Convenient online submission

- Rigorous peer review

- Open access: articles freely available online

High visibility within the field

- Retaining the copyright to your article

Submit your next manuscript at $>$ springeropen.com 